\title{
Association between obstructive sleep apnea (OSA) and depression and the effect of continuous positive airway pressure (CPAP) treatment
}

\author{
This article was published in the following Dove Press journal: \\ Neuropsychiatric Disease and Treatment \\ 14 December 201 I \\ Number of times this article has been viewed
}

\author{
Amr Makram El-Sherbini' \\ Adel Salah Bediwy ${ }^{2}$ \\ Ashraf El-Mitwalli ${ }^{3}$ \\ 'Department of Psychiatry, Elminia \\ University Hospital, Faculty \\ of Medicine, Elminia, ${ }^{2}$ Chest \\ Department, Faculty of Medicine, \\ Tanta University, ${ }^{3}$ Department \\ of Neurology, Mansoura School \\ of Medicine, University of Mansoura, \\ Mansoura, Egypt
}

Background: Obstructive sleep apnea (OSA) is a relatively common disorder which has a negative impact on the psychological well-being of affected individuals.

Objective: To assess the association between OSA and depression as well as the effect of treatment with continuous positive airway pressure (CPAP).

Methods: A total of 37 newly diagnosed individuals with OSA underwent an overnight polysomnography and were assessed using the Epworth Sleepiness Scale (ESS), the Hamilton Depression Rating Scale (HDRS), and the Structured Clinical Interview for Diagnostic and Statistical Manual of Mental Disorders, Fourth Edition. Patients were assessed before and after 2 months of CPAP use.

Results: Of the 37 patients included in the study, 21 (56.7\%) had clinically relevant depression as indicated by a score $>10$ on the HDRS and eleven patients $(29.7 \%)$ met the diagnostic criteria for a major depressive episode using the Structured Clinical Interview. Scores on the HDRS were correlated with the Apnea Hypoxia Index, ESS scores, and oxygen saturation. Patients showed a significant reduction in depressive symptoms and improvement in ESS scores after CPAP treatment.

Conclusion: Patients with OSA should be screened carefully for depressive disorders. CPAP should be tried first before starting other treatment modalities for depression.

Keywords: obstructive sleep apnea, depression in OSA, CPAP and depression

\section{Introduction}

Obstructive sleep apnea (OSA) is by far the most common form of sleep-disordered breathing, and is defined as frequent episodes of obstructed breathing during sleep. Specifically, it is characterized by sleep-related decreases (hypopneas) or pauses (apneas) in respiration. An obstructive apnea is defined as at least a 10-second interruption of oronasal airflow, corresponding to a complete obstruction of the upper airways despite continuous chest and abdominal movements, and is associated with a decrease in oxygen saturation and/or arousals from sleep. It is confirmed by polysomnography recording determining an apnea-hypopnea index (AHI) of $>5$ per hour of sleep. ${ }^{1}$

The relation between OSA and depression has been the subject of many studies. ${ }^{2-5}$ Most reported increased levels of depression in individuals with OSA. ${ }^{2,3}$ Studies that found an increased rate of depression in patients with OSA, also found that the prevalence of depression was variable; the rate was $17.6 \%$ using the Diagnostic and Statistical Manual of Mental Disorders, Fourth Edition (DSM-IV) criteria. ${ }^{4}$ When the research
Correspondence: Adel Salah Bediwy Chest Department, Faculty of Medicine, Tanta University, Egypt

Tel +2010056II5I5, +97339240642

Email adelsalahI@yahoo.com 
diagnostic criteria for an affective disorder was used, the rate was around $40 \%,{ }^{5}$ whereas Millman et $\mathrm{al}^{6}$ observed that $45 \%$ of their OSA patients had depressive symptoms on the Zung Self-Rating Depression Scale, and another study found that $58 \%$ of individuals with OSA fulfilled the Diagnostic and Statistical Manual of Mental Disorders, Third Edition criteria for major depression. ${ }^{7}$ The use of different tools for diagnosing depression, with different criteria, cutoff points, and illness durations, may account for some of the variations in reported results in different studies. ${ }^{2-5}$ Alternatively, some studies found no such association. ${ }^{8-10}$ In addition, many patients with OSA are diagnosed as having a depressive disorder and many receive antidepressant medications before they are referred for assessment for sleep disorder. ${ }^{11}$ Thus, some researchers argue for a causal relation, with sleep fragmentation in OSA causing depression, ${ }^{12,13}$ others argue that a common neurobiological mechanism is the underlying cause for this association, with serotonin playing a central role, ${ }^{13,14}$ and yet another group claim that no clear relationship exists between the two disorders. ${ }^{15}$

Several studies attempted to delineate the relation between depression and components of OSA, either those observed clinically such as excessive daytime sleepiness ${ }^{16}$ or those components detected on polysomnography such as AHI, ${ }^{17,18}$ oxygen saturation, and sleep stages ${ }^{15}$ and arousals detected by electroencephalogram (EEG). Results of those studies are quite variable with some studies finding correlations between some of the mentioned variables and depression, but others failing to find such an association. ${ }^{15-17}$ Still more research in this field is needed to adequately delineate the significance of such a relation.

The use of continuous positive air pressure (CPAP) is the treatment of choice for OSA. However, studies to date on its effect on depressive symptoms have yielded inconsistent findings. Most of the studies reported a positive effect of CPAP on the psychological well-being of individuals treated in this way, ${ }^{6,19,20}$ while others reported no such improvement. ${ }^{21,22}$

The use of CPAP is the treatment of choice for OSA. However, studies to date on its effect on depressive symptoms have yielded inconsistent findings.

\section{Objective}

In this study, it was aimed to determine the rate of depression associated with OSA, and to study the effect of CPAP on the psychological condition of these patients.

\section{Methods}

Individuals were recruited from the sleep laboratory of the Hospital of Bahrain. All patients who met the standard indications for polysomnographic evaluation for the suspected diagnosis of OSA were eligible for entry into the study. The local ethics committee approved the study and patients who agreed to participate in the study gave informed consent. Patients with a previous psychiatric diagnosis or who were on antidepressant medications were excluded from the study. Patients with chronic diseases (respiratory, renal, cardiac, neurological, and hepatic disorders) were also excluded.

All patients underwent overnight polysomnography using the Alice ${ }^{\circledR} 5$ Polysomnography System (Philips Respironics, Murraysville, PA) with Alice Sleepware Software. This polysomnogram consisted of overnight recording of left and right electrooculogram, chin electromyogram, left and right tibialis anterior electromyogram, central and occipital EEG, electrocardiogram, airflow measurement, body position sensor, snoring sensor and microphone, respiratory effort belt (abdominal and thoracic), and pulse oximetry. The sleep study began around $11 \mathrm{pm}$ and the duration of the study was at least 5 hours. The patients were instructed to avoid sleeping during the daytime before the study and to avoid alcohol, sedatives, hypnotics, and other medicines affecting sleep.

Patients with OSA were interviewed and assessed using three tools.

1. The Epworth Sleepiness Scale was used to assess daytime sleepiness. Patients rated the likelihood of falling asleep in eight specific situations on a $0-3$ scale, with 0 meaning no chance at all of falling asleep, and 3 representing a high chance of falling asleep. Thus, the scale goes from 0 to 24 . A score of ten suggests excessive daytime sleepiness. ${ }^{23}$

2. The Hamilton Depression Rating Scale (HDRS) is an inventory employed to detect and identify the intensity or severity of the signs and symptoms of depression; 17 questions are divided into two categories. The first category consists of nine questions scored on a five-point scale; the second category consists of eight questions rated on a three-point scale. A total score is computed reflecting the degree of symptom severity. A score $>10$ indicates impairment. ${ }^{24}$

3. The Structured Clinical Interview for DSM-IV (SCID) is a semistructured interview used to establish the major Axis I diagnoses in DSM-IV. It is administered by a clinician and includes an introductory overview followed by nine modules, seven of which represent the major Axis I diagnostic classes. Only the section on mood disorders was used. ${ }^{25}$

Patients were assessed twice: a baseline was done immediately after the sleep study and confirmation of the presence 
of OSA, and a second assessment was completed 2 months after treatment with the CPAP. Polysomnography was done and interpreted by both the pulmonologist and neurologist, and the psychiatrist was blinded to the severity of sleep disturbance while conducting psychiatric assessment.

Data were analyzed using SPSS (v 15.0; IBM Corp, Armonk, NY). Means with standard deviations or percentages were used to describe the sample. Group differences were assessed with unpaired $t$-tests and the changes after treatment were assessed with paired $t$-tests. Comparison for categorical variables was done using the chi-square test. The Pearson linear correlation was also determined between certain variables. A $P$ value $<0.05$ was considered to indicate statistical significance.

\section{Results}

From June 2010 to December 2010, 44 patients were diagnosed with OSA and were eligible to be included in the study. Seven patients were excluded from the study: two refused to contribute in the study, two had a previous psychiatric diagnosis, two refused treatment with CPAP, and one was not compliant with CPAP. The remaining 37 patients were included in the study.

The mean age of individuals was $44.9 \pm 8.8$; there was no statistical difference between males and females as regards the age. Twenty-one individuals had HDRS scores above the cut-off point of the scale of ten (indicating some sort of depression), 13 individuals (35.1\%) had mild depressive symptoms while four had moderate symptoms (10.8\%), and the same number had severe symptoms. It is important to note that the levels of depressive symptoms obtained from the Hamilton scale are a continuous measure of depressive symptoms and do not necessarily always equate with a formal categorical diagnosis of major depressive disorder. The mean total score of the HDRS was $11.5 \pm 9.6$, females had statistically significant higher scores than males $(P=0.049)$. Using the SCID, eleven patients fulfilled the clinical criteria of a major depressive disorder according to DSM-IV, seven with mild and four with moderate depressive episodes. The mean ESS was 11.6 \pm 8.6 while the mean AHI was 27.7 \pm 7.3 ; there was no statistically significant difference between males and females on either parameter. The mean arousal index was $33.4 \pm 5.5$ as indicated by EEG analysis of the sleep stages and there was no statistically significant difference between males and females. The lowest oxygen saturation $\left(\mathrm{SaO}_{2}\right)$ during the sleep study showed a mean of $67.5 \% \pm 3 \%$ and there was no statistically significant difference between males and females (Table 1).

The age of the patient correlated positively with AHI $(P=0.000)$, and ESS $(P=0.02)$ and lowest oxygen saturation $(P=0.048)$. There was a significant negative correlation between the lowest oxygen saturation and HDRS score $(P=0.000)$. ESS was correlated positively with the HDRS $(P=0.000)$ and so was the AHI $(P=0.000)$ (Table 2$)$.

There was a statistically significant decrease in the HDRS in all groups when patients with OSA used the CPAP for 2 months (Table 3). Six of the eleven patients previously diagnosed with a depressive episode by the SCID prior to

Table I Patient's description on first examination

\begin{tabular}{|c|c|c|c|c|}
\hline & Males $(\mathbf{N}=24)$ & Females $(\mathbf{N}=13)$ & Total $(\mathbf{N}=37)$ & Difference \\
\hline Age & Mean $=45.3 \pm 9.2$ & Mean $=44.2 \pm 8.3$ & Mean $=44.9 \pm 8.8$ & $t=0.3, P=0.7$ \\
\hline \multicolumn{5}{|l|}{ HDRS } \\
\hline Normal (0-9) & $12(50 \%)$ & $4(30.8 \%)$ & $16(43.3 \%)$ & chi-square $=1.7, P=0.7$ \\
\hline Mild (10-13) & $8(33.3 \%)$ & $5(38.5)$ & $13(35.1)$ & \\
\hline Moderate (14-7) & $2(8.3 \%)$ & $2(15.4 \%)$ & $4(10.8)$ & \\
\hline Severe $(>17)$ & $2(8.3 \%)$ & $2(15.4 \%)$ & $4(10.8 \%)$ & \\
\hline Total score & Mean $=9.3 \pm 7.5$ & Mean $=15.6 \pm 9.8$ & Mean $=11.5 \pm 9.6$ & $t=2 . \mathrm{I}, \mathrm{DF} 35, P=0.049 *$ \\
\hline SCID & & & & chi-square $=0.8, P=0.7$ \\
\hline No disorder & 18 (75\%) & $8(61 \%)$ & $26(70.3)$ & \\
\hline Mild depression ${ }^{\mathrm{a}}$ & $4(16.7 \%)$ & $3(23 \%)$ & 7 (I8.9\%) & \\
\hline Mod depression ${ }^{\mathrm{b}}$ & $2(8.4 \%)$ & $2(15 \%)$ & $4(10.8)$ & \\
\hline ESS & Mean $=10.9 \pm 7.5$ & Mean $=13.1 \pm 9.8$ & Mean $=11.6 \pm 8.6$ & $t=0.26, P=0.1$ \\
\hline $\mathrm{AHI}$ & Mean $=28.2 \pm 6.9$ & Mean $=26.9 \pm 8.2$ & Mean $=27.7 \pm 7.3$ & $t=0.5, \mathrm{DF} 35, P=0.6$ \\
\hline Lowest $\mathrm{O}_{2}$ saturation $\left(\mathrm{SaO}_{2}\right)$ & Mean $=67.2 \pm 2.8$ & Mean $=67.6 \pm 3.3$ & Mean $=67.5 \pm 3$ & $t=0.3, \mathrm{DF} 35, P=0.7$ \\
\hline Arousal index & Mean $=33.8 \pm 4.8$ & Mean $=32.7 \pm 6.9$ & Mean $=33.45 \pm 5.5$ & $t=0.5, \mathrm{DF} 35, P=0.6$ \\
\hline
\end{tabular}

Notes: There was no statistical difference between the age of males and females. Regarding the mean total score of the HDRS, females had statistically significant higher scores than males. Patient assessment using SCID revealed no significant difference between males and females. ESS, AHI, lowest $\mathrm{O}_{2}$ saturation, and arousal index showed no statistically significant difference between males and females. ${ }^{a}$ Mild depression = major depressive episode, mild; ${ }^{b}$ mod depression $=$ major depressive episode, moderate; *significant.

Abbreviations: AHI, apnea-hypopnea index; DF, degree of freedom; ESS, Epworth Sleepiness Score; HDRS, Hamilton Depression Rating Scale; SCID, Structured Clinical Interview for Diagnostic and Statistical Manual of Mental Disorders, Fourth Edition. 
Table 2 Correlation studies at initial assessment

\begin{tabular}{|c|c|c|c|c|}
\hline & HDRS & AHI & ESS & Lowest $\mathrm{SaO}_{2}$ \\
\hline Age & $r=0.25, P=0.1$ & $r=0.6, P=0.000^{*}$ & $r=0.4, P=0.02^{*}$ & $r=0.3, P=0.048^{*}$ \\
\hline Arousal index & $r=0.04, P=0.4$ & $r=0.06, P=0.5$ & $r=0.07, P=0.6$ & $r=0.05, P=0.7$ \\
\hline Lowest oxygen saturation & $r=-0.8, P=0.000 *$ & $r=-0.8, P=0.000 *$ & $r=-0.7, P=0.000 *$ & \\
\hline ESS & $r=0.9, P=0.000^{*}$ & $r=0.9, P=0.000^{*}$ & & \\
\hline $\mathrm{AHI}$ & $r=0.7, P=0.000^{*}$ & & & \\
\hline
\end{tabular}

Note: *Significant.

Abbreviations: AHI, apnea-hypopnea index; ESS, Epworth Sleepiness Scale; HDRS, Hamilton Depression Rating Scale.

use of the CPAP, no longer met the diagnostic criteria needed for the diagnosis of a depressive episode after CPAP use and only five patients met the diagnosis of a mild depressive episode. The ESS scores decreased from a mean of $11.6 \pm 8.6$ to $5.1 \pm 3.1$ and this difference was statistically significant $(t=7.6, P=0.000)$. ESS scores showed a significant positive correlation with HDRS after the use of CPAP $(\mathrm{r}=0.7$, $P=0.000)$.

The changes in individual symptoms on HDRS after the use of CPAP are given in Table 4; the scores for most of the items on HDRS decreased significantly. In addition, a separate analysis for HDRS scores was conducted, excluding four items: three concerned with sleep and one with fatigue. The aim was to determine the change in the HDRS scores when removing symptoms that could be caused by sleep apnea itself (Table 5).

\section{Discussion}

In this study, it was aimed to determine the rate of depressive symptoms associated with OSA, and the relation between different variables of sleep apnea with depression, as well as to study the effect of CPAP on the psychological well-being of these patients. Over a period of 6 months, the number of male patients referred for assessment for possible OSA was nearly twice that of females ( 24 males compared with 13 females). This discrepancy might reflect the increased incidence of OSA in males reported in several studies ${ }^{26,27}$ but could also be due to bias in referral with more male patients referred for the sleep study. In the current study, the main aim was not to determine the prevalence of the disorder in a specific population but rather to study the association between OSA and depression.

More than half of the studied sample (56.7\%) had depressive symptoms as measured by HDRS; eight of these patients $(21.6 \%)$ had clinically significant moderate to severe symptoms. This high prevalence is in line with findings reported in some studies in which OSA was highly associated with depression. ${ }^{6-8}$ Females showed a higher rate of depression although this difference was not statistically significant; females had statistically higher scores than males on the HDRS, and this finding has been reported in previous studies. ${ }^{9}{ }^{15}$ When using the SCID to establish a DSM-IV diagnosis, the total number of individuals with a clinical diagnosis of depressive disorder was eleven (29.7\%), and there was no statistically significant difference between females and males in the rate of occurrence of depressive disorders using this method. From the above, it can be concluded that the prevalence of depression in the same sample varied according to the tool used, with a high prevalence when measured with the HDRS, a tool used mainly to measure the severity of depressive symptoms rather than to generate a diagnosis. This finding can explain some of the differences in the rate of depression in individuals with OSA found across different studies. ${ }^{2-5}$ It can also be concluded that the main difference between females and males might be in the degree of severity of depressive symptoms rather than the mere prevalence of depression in individuals with OSA.

Table 3 Comparison between patients on HDRS before and after CPAP treatment

\begin{tabular}{|c|c|c|c|c|}
\hline & Individuals (N) & HDRS at baseline & HDRS at follow-up & Differences \\
\hline Mild & 13 & Mean II.9 \pm 0.8 & Mean $7 \pm 1.9$ & $t=11.8, \mathrm{DF} 12, P=0.000^{*}$ \\
\hline Moderate & 4 & Mean $15.7 \pm 0.9$ & Mean II $.4 \pm 0.9$ & $t=4.3, \mathrm{DF} 3, P=0.02 *$ \\
\hline Severe & 4 & Mean $35.5 \pm 3.5$ & Mean $27.2 \pm 3.4$ & $t=3, \mathrm{DF} 3, P=0.047^{*}$ \\
\hline Total & 21 & Mean II.5 \pm 9.6 & Mean $7.4 \pm 7.8$ & $t=8.6, \mathrm{DF} 36, P=0.000^{*}$ \\
\hline Normal & 16 & Mean $4.3 \pm 2.5$ & Mean $1.8 \pm 1$ & $t=5, \mathrm{DF}$ I5, $P=0.000^{*}$ \\
\hline
\end{tabular}

Note: *Significant.

Abbreviations: CPAP, continuous positive airway pressure; DF, degree of freedom; HDRS, Hamilton Depression Rating Scale. 
Table 4 Changes in individual symptoms on HDRS after CPAP use

\begin{tabular}{|c|c|c|c|}
\hline & Before CPAP (baseline) & After CPAP & Difference \\
\hline Depressed mood & Mean $=1.7$, SD I.I & Mean $=$ I, SD 0.7 & $t=6.9, \mathrm{DF}=36, P=0.000$ \\
\hline Feeling of guilt & Mean $=0.7$, SD 0.9 & Mean $=0.5$, SD 0.6 & $t=3.2, \mathrm{DF}=36, P=0.000$ \\
\hline Suicide & Mean $=0.2$, SD 0.5 & Mean $=0.1$, SD 0.3 & $t=1.7, \mathrm{DF}=36, P=0.08$ \\
\hline Work and activities & Mean $=1.3$, SD I.I & Mean $=0.9$, SD 0.8 & $t=4.9, \mathrm{DF}=36, P=0.000$ \\
\hline Retardation & Mean $=0.7$, SD 0.9 & Mean $=0.5$, SD 0.8 & $t=2.3, \mathrm{DF}=36, P=0.02$ \\
\hline Agitation & Mean $=0, S D$ & Mean $=0, S D$ & Not calculated \\
\hline Anxiety - psychic & Mean $=0.5$, SD I.I & Mean $=0.4$, SD 0.9 & $t=1.6, \mathrm{DF}=36, P=0.1$ \\
\hline Anxiety - somatic & Mean $=0.4$, SD 0.9 & Mean $=0.3$, SD 0.8 & $t=2, \mathrm{DF}=36, P=0.04$ \\
\hline Somatic symptoms - GI & Mean $=0.1$, SD 0.5 & Mean $=0.1$, SD 0.5 & Not calculated \\
\hline Genital symptoms & Mean $=0.5$, SD 0.7 & Mean $=0.2$, SD 0.6 & $t=3 . \mathrm{I}, \mathrm{DF}=36, P=0.003$ \\
\hline Hypochondrias & Mean $=0.3$, SD 0.7 & Mean $=0.1$, SD 0.5 & $t=1.9, \mathrm{DF}=36, P=0.05$ \\
\hline Weight loss & Mean $=0.6$, SD 0.6 & Mean $=0.5$, SD 0.6 & $t=3 . \mathrm{I}, \mathrm{DF}=36, P=0.003$ \\
\hline Insight & Mean $=0, S D$ & Mean $=0, \mathrm{SD}$ & Not calculated \\
\hline Insomnia - early & Mean $=0.7$, SD 0.7 & Mean $=0.5$, SD 0.5 & $t=2.5, \mathrm{DF}=36, P=0.0 \mathrm{I}$ \\
\hline Insomnia - middle & Mean $=1.3$, SD 0.5 & Mean $=0.7$, SD 0.6 & $t=6, \mathrm{DF}=36, P=0.000$ \\
\hline Insomnia - late & Mean $=0.5$, SD 0.6 & Mean $=0.2$, SD 0.5 & $t=3.4, \mathrm{DF}=36, P=0.002$ \\
\hline Somatic symptom general (fatigue) & Mean $=1.5$, SD 0.6 & Mean $=0.9$, SD 0.6 & $t=6.7, \mathrm{DF}=36, P=0.000$ \\
\hline
\end{tabular}

Note: *Statistically significant $(P \leq 0.05)$.

Abbreviations: CPAP, continuous positive airway pressure; DF, degree of freedom; HDRS, Hamilton Depression Rating Scale.

There was a significant negative correlation between lowest oxygen saturation and HDRS scores. This finding was reported by Means et al, ${ }^{28}$ but McCall et al ${ }^{15}$ reported the inverse relation for females with OSA; the difference between the present authors' results and their results might be due to gender differences of the examined population. A positive correlation was found between daytime sleepiness - as measured by the ESS - and HDRS, and this finding has been reported in some studies. ${ }^{16,29}$ The AHI was correlated positively with the HDRS scores and this finding has also been reported by some studies, ${ }^{6,15}$ although Dominici and Gomes ${ }^{18}$ did not find such a relation. The difference in reported results might be due to methodological differences, particularly the use of a different tool to assess depression the Beck Depression Inventory - with a different cut-off point and different variables assessed. Thus, the severity of depression was correlated with components of OSA and this finding adds to the hypothesis that a causal relation might exist between the two disorders.

At follow-up, marked improvement of depression was found after the use of CPAP, as reflected by statistically significant improvement on the HDRS. In addition, only five patients of those diagnosed with the SCID as having a depressive disorder still met the DSM-IV diagnostic criteria for a depressive episode after CPAP treatment. These patients might require specific antidepressant treatment whereas the remaining six patients would not require additional treatment with CPAP. Most studies showed improvement of depressive symptoms with the use of the CPAP, ${ }^{6,19,20}$ and this finding is replicated in the current study, but some studies ${ }^{21,22}$ found no improvement in depression after CPAP use. In the first study ${ }^{21}$ only individuals with severe OSA were included and the second study included a limited number of patients.22 The difference in results might also be due to differences in the tool used and cut-off points taken and this is clear in the current study. When HDRS was used as the tool to assess depression severity the improvement after CPAP use was very obvious but, when SCID was used, improvement was less obvious. In addition, when the scores for symptoms of fatigue and insomnia, which can be caused by OSA, were removed and then the total scores of the HDRS were calculated, the scores significantly dropped. Thus tools may vary in their

Table 5 HDRS scores after removal of sleep and fatigue symptoms

\begin{tabular}{|c|c|c|c|}
\hline & Baseline & After CPAP & Difference \\
\hline HDRS total score & Mean $=11.5$, SD 9.6 & Mean $=7.5$, SD 7.8 & $t=8.6, \mathrm{DF}=36, P=0.000$ \\
\hline HDRS score omitting fatigue and insomnia & Mean $=7.4$, SD 8 & Mean $=5$, SD 6.5 & $t=6.6, \mathrm{DF}=36, P=0.000$ \\
\hline Difference & $t=13.1, \mathrm{DF}=36, P=0.000$ & $t=8.7, \mathrm{DF}=36, P=0.000$ & - \\
\hline
\end{tabular}

Note: *Statistically significant $(P \leq 0.05)$.

Abbreviations: CPAP, continuous positive airway pressure; DF, degree of freedom; HDRS, Hamilton Depression Rating Scale. 
ability to detect changes in depressive symptoms according to the items screened by that tool which may account for some differences reported in different studies. ${ }^{2-5}$

In the current study, a statistically significant correlation between ESS score and HDRS scores on follow-up indicated that as daytime sleepiness improves, depression improves. This finding has been reported in previous studies ${ }^{30,31}$ and supports the hypothesis that sleep fragmentation and daytime sleepiness might have an important role in the generation of depression in these individuals.

Analysis of individual symptoms on HDRS showed statistically significant improvement in core depressive symptoms after CPAP use; this indicates a favorable effect of CPAP on depressive symptoms. The authors of the present study also conducted analysis comparing the total HDRS score with the total score after removing the scores of four items concerning insomnia and fatigue, which could be caused by sleep apnea itself (Table 5). The results of this analysis showed statistically significant differences between the two scores with a marked decrease in scores after removal of these four symptoms. However, changes occurring in both scores after using the CPAP remained statistically significant. Depression in OSA can be overdiagnosed using tools to screen for depression. However, changes in depressive psychopathology after CPAP use can still be detected by these instruments in patients with OSA.

This study has its own limitations, particularly the limited number of patients included and the unequal number of males and females.

In conclusion, depression was prevalent in individuals with OSA and was more severe in females. In addition, depression was highly correlated with variables of OSA, and improvement in OSA with the use of CPAP was associated with improvement in severity of depression. Careful assessment for OSA in patients presenting with depressive symptoms is important to avoid unnecessary use of antidepressant medications because a large subset of those patients will improve clinically using the CPAP only.

\section{Disclosure}

The authors have no conflicts of interest to declare in relation to this work.

\section{References}

1. American Academy of Sleep Medicine. International Classification of Sleep Disorders: Diagnostic and Coding Manual. 2nd ed. Westchester, IL: American Academy of Sleep Medicine; 2005.
2. Sanchez AI, Martnez P, Miro E, Baedwell WA, Buela-Casal G. CPAP and behavioral therapies in patients with obstructive sleep apnea: effects on daytime sleepiness, mood, and cognitive function. Sleep Med Rev. 2009; 13:223-233.

3. Harris M, Glozier N, Ratnavadivel R, Grunstein RR. Obstructive sleep apnea and depression. Sleep Med Rev. 2009;13:437-444.

4. Ohayon MM. The effects of breathing-related sleep disorders on mood disturbances in the general population. J Clin Psychiatry. 2003;64:1195-1200.

5. Reynolds CF, Kupfer DJ, McEachran AB, Taska LS, Sewitch DE, Coble PA. Depressive psychopathology in male sleep apneics. J Clin Psychiatry. 1984;45:287-290.

6. Millman RP, Fogel BS, McNamara ME, Carlisle CC. Depression as a manifestation of obstructive sleep apnea: reversal with nasal continuous positive airway pressure. J Clin Psychiatry. 1989;50:348-351.

7. Mosko S, Zetin M, Glen S, et al. Self-reported depressive symptomatology, mood ratings, and treatment outcome in sleep disorders patients. J Clin Psychol. 1989;45:51-60.

8. Phillips BA, Berry DT, Lipke-Molby TC. Sleep-disordered breathing in healthy, aged persons: fifth and final year follow-up. Chest. 1996;110:654-658.

9. Pillar G, Lavie P. Psychiatric symptoms in sleep apnea syndrome: effects of gender and respiratory disturbance index. Chest. 1998;114: 697-703.

10. Bardwell WA, Berry CC, Ancoli-Israel S, Dimsdale JE. Psychological correlates of sleep apnea. J Psychosom Res. 1999;47:583-596.

11. Stores G. Misdiagnosing sleep disorders as primary psychiatric conditions. Adv Psychiatr Treat. 2003;9:69-77.

12. Schröder CM, O’Hara R. Depression and Obstructive Sleep Apnea (OSA). Ann Gen Psychiatry. 2005;4:13.

13. Adrien J. Neurobiological bases for the relation between sleep and depression. Sleep Med Rev. 2002;6:341-351.

14. Veasey SC. Serotonin agonists and antagonists in obstructive sleep apnea: therapeutic potential. Am J Respir Med. 2003;2:21-29.

15. McCall WV, Harding D, O'Donovan C. Correlates of depressive symptoms in patients with obstructive sleep apnea. J Clin Sleep Med. 2006;2:424-426.

16. Kawahara S, Akashiba T, Akahoshit T, Horie T. Nasal CPAP improves the quality of life and lessens the depressive symptoms in patients with obstructive sleep apnea syndrome. Intern Med. 2005;44:422-427.

17. Kjelsberg FN, Ruud EA, Stavem K. Predictors of symptoms of anxiety and depression in obstructive sleep apnea. Sleep Med. 2005;6: 341-346.

18. Dominici M, Gomes MD. Obstructive sleep apnea (OSA) and depressive symptoms. Arq Neuropsiquiatr. 2009;67:35-39.

19. Jenkinson C, Davis RJ, Mullins R, Stradling JR. Comparison of therapeutic and subtherapeutic nasal continuous positive airway pressure for obstructive sleep apnoea: a randomised prospective parallel trial. Lancet. 1999;353:2100-2105.

20. Schwartz DJ, Kohler WS, Karatinos G. Symptoms of depression in individuals with obstructive sleep apnea may be amenable to treatment with continuous positive airway pressure. Chest. 2005;128:1304-1309.

21. Borak J, Clieslicki JK, Kozieji M, Matusezewski A, Zielinski J. Effects of CPAP treatment on psychological status in patients with severe obstructive sleep apnea. J Sleep Res. 1996;5:123-127.

22. Klonoff H, Fleetham J, Taylor DR, Clark C. Treatment outcome of obstructive sleep apnea: physiological and neuropsychological concomitants. J Nerv Ment Dis. 1987;175:208-212.

23. Johns MW. A new method for measuring daytime sleepiness: the epworth sleepiness scale. Sleep. 1991;14:540-545.

24. Hamilton M. Development of a rating scale for primary depressive illness. Br J Soc Clin Psychol. 1967;6:278-296.

25. First MB, Gibbon M, Spitzer RL, Williams JBW. Structured Clinical Interview for DSM-IV Axis I Disorders, Clinician Version (SCID-CV). Washington, DC: American Psychiatric Press, Inc; 1996. 
26. Young T, Palta M, Dempsey J, Skatrud J, Weber S, Badr S. The occurrence of sleep-disordered breathing among middle-aged adults. N Engl J Med. 1993;328:1230-1235.

27. Ancoli-Israel S, Kripke DF, Klauber MR, Mason WJ, Fell R, Kaplan O. Sleep-disordered breathing in community-dwelling elderly. Sleep. 1991;14:486-495.

28. Means MK, Lichstein KL, Edinger JD, et al. Changes in depressive symptoms after continuous positive airway pressure treatment for obstructive sleep apnea. Sleep Breath. 2003;7:31-42.
29. Rosenberg R, Doghramji P. Optimal treatment of obstructive sleep apnea and excessive sleepiness. Adv Ther. 2009;26:295-312.

30. Koutsourelakis I, Perraki E, Economou NT, et al. Predictors of residual sleepiness in adequately treated obstructive sleep apnoea patients. Eur Respir J. 2009;34:687-693.

31. Dixon JB, Dixon ME, Anderson ML, Schachter L, O’Brien PE. Daytime sleepiness in the obese: not as simple as obstructive sleep apnea. Obesity. 2007;15:2504-2511.

\section{Publish your work in this journal}

Neuropsychiatric Disease and Treatment is an international, peerreviewed journal of clinical therapeutics and pharmacology focusing on concise rapid reporting of clinical or pre-clinical studies on a range of neuropsychiatric and neurological disorders. This journa is indexed on PubMed Central, the 'PsycINFO' database and CAS
The manuscript management system is completely online and includes a very quick and fair peer-review system, which is all easy to use. Visit http://www.dovepress.com/testimonials.php to read real quotes from published authors.

Submit your manuscript here: http://www.dovepress.com/neuropsychiatric-disease-and-treatment-journal 